\title{
Immigrants: Big Challenge and Silent Threat to Implement Malaria Elimination Program in Hormozgan Province, Iran
}

\author{
Golsoom Rashid (iD) ${ }^{1}$, Aboozar Soltani (iD ${ }^{2}$, Ebrahim Eftekhar (iD ${ }^{3}$, Reza Shafiei (i) ${ }^{4}$ and Habibollah Turki \\ (ii) ${ }^{1}{ }^{*}$ \\ ${ }^{1}$ Infectious and Tropical Diseases Research Center, Hormozgan Health Institute, Hormozgan University of Medical Sciences, Bandar Abbas, Iran \\ ${ }^{2}$ Research Center for Health Sciences, Institute of Health, Department of Medical Entomology and Vector Control, School of Health, Shiraz University of Medical Sciences, \\ Shiraz, Iran \\ ${ }^{3}$ Molecular Medicine Research Center, Hormozgan Health Institute, Hormozgan University of Medical Sciences, Bandar Abbas, Iran \\ ${ }^{4}$ Vector-Borne Diseases Research Center, North Khorasan University of Medical Sciences, Bojnurd, Iran \\ "Corresponding author: Infectious and Tropical Diseases Research Center, Hormozgan Health Institute, Hormozgan University of Medical Sciences, Bandar Abbas, Iran. Email: \\ h.turki@hums.ac.ir
}

Received 2019 November 26; Revised 2020 February 25; Accepted 2020 February 25.

\section{Abstract}

Background: Detection and monitoring of asymptomatic malaria cases, especially among immigrants, are the main parts of the successful malaria elimination program. To achieve this aim, a sensitive detection method is required.

Objectives: This study aimed to investigate immigrant asymptomatic malaria cases in Bandar Abbas district using microscopic, Rapid Diagnosis Test (RDT), and Nested PCR.

Methods: In this cross-sectional study, 330 blood samples of immigrants living in Bandar Abbas were collected and their status in terms of Plasmodium infection was assessed using microscopic, RDT and Nested-PCR techniques.

Results: Five positive cases were found when molecular analysis was applied. However, microscopy and RDT methods showed no positive malaria cases.

Conclusions: The results of this study are indicative of a big challenge to the malaria elimination program posed by presence of asymptomatic cases who migrated from malaria endemic areas. In addition, accurate diagnosis of asymptomatic malaria cases requires the use of molecular methods.

Keywords: Asymptomatic Malaria, Immigrants, Plasmodium, Malaria Elimination

\section{Background}

Malaria is one of the most common blood infectious and tropical diseases worldwide that is considered by WHO programs. It is caused by protozoan parasite of the Plasmodium sp., which is transmitted by female anopheles mosquito bite. Human malaria is caused by five species of Plasmodium: P. vivax is a dominant species in the world and Iran, P. falciparum is related to the highest mortality, P. ovale and $P$. malariae generally cause a mild form of the disease, $P$. knowlesi are zoonotic malaria and rarely cause disease in humans (1). Despite the decline in malaria cases in recent years, the disease remains a major health problem in the tropical and subtropical regions of the world $(2,3)$.

The WHO has reported that in 2017, about 219 million malaria cases were recorded worldwide of which 435,000 have died. According to this report, malaria transmission is ongoing in 87 countries (4). Malaria is considered the most important parasitic disease in Iran and the ma- jor health problem in the southern and southeastern regions of this country. Following the successful program of the malaria control and a significant reduction in the incidence of malaria transmission, the first phase of the malaria elimination program supported by the WHO was started in 2010 (1). In 2019, the positive cases of malaria in Bandar Abbas county have been reported to be 8 , all of which were $P$. vivax imported from other countries (Vice Chancellor for Health, Bandar Abbas University of Medical Sciences, unpublished data).

The malaria elimination program is ongoing in the province of Hormozgan. The proper weather conditions and abundance of anopheles mosquitoes make it potential for the vector to transmit malaria throughout all seasons. Anopheles stephensi is the most important carrier in this area (5). The main goal of the malaria elimination program is to stop the local transmission, but there are challenges in this program, so in the presence of parasitic reser- 
voirs and abundant carriers, local transmission of malaria is possible. On the other hand due to the presence of immigrants from malaria endemic countries, which may play a main role as the asymptomatic parasitic reservoir of malaria in the transmission cycle, the need exists for monitoring and effective tools for identifying and detecting asymptomatic malaria cases in foreign nationals. Although a large number of studies have been conducted in Iran to diagnose asymptomatic malaria cases using molecular methods in indigenous people from malaria endemic areas (6-12), few studies have been conducted on immigrants. In two studies on immigrants by Nateghpour et al. in Sistan and Baluchestan and Amirshekari et al. in Kerman province, some positive asymptomatic cases were identified among immigrants from Afghan and Pakistani nationalities $(13,14)$.

There are a number of published studies in other countries investigating immigrants as asymptomatic malaria cases. In these studies, researchers attempted to evaluate the role of immigrants in malaria transmission in Russia, Bulgaria, United States and Canada (15-18). The key aim of this research was to monitor and detect immigrant's asymptomatic malaria cases in Bandar Abbas. Given that the routine diagnostic techniques (microscopy and Rapid Diagnosis Test (RDT)) don't have enough sensitivity to detect asymptomatic malaria cases, a robust and sensitive molecular method was applied in this study. Certainly, the outcomes of current research contribute to the successful implementation of the malaria elimination program in Hormozgan province and Iran.

\section{Objectives}

This study was aimed to investigate immigrant asymptomatic malaria cases in Bandar Abbas district using microscopic, rapid diagnosis test (RDT), and Nested PCR.

\section{Methods}

\subsection{Study Area}

This study was performed to identify asymptomatic malaria cases among immigrants in Bandar Abbas with emphasis on using a high sensitive molecular method. Bandar Abbas as an important area for malaria transmission was selected to investigate the presence of asymptomatic malaria cases among refugees. This area was selected in accordance with the National Malaria Elimination Program and in coordination with the Center for Malaria Prevention and Control related to the Ministry of Health. Bandar Abbas, the capital of Hormozgan province, is a port located on the southern coast of Iran with hot desert climate and temperature ranged $5-49^{\circ} \mathrm{C}$ and average relative humidity of $65 \%$. Bandar Abbas is situated on flat ground with an average altitude of 9 meters (30 ft) above the sea level (Figure 1).

\subsection{Study Population and Inclusion Criteria}

In this cross-sectional study a total of 330 cases $(18.3 \%$ female and $81.7 \%$ male) who were registered in refugee camps, enrolled in the study (Table 1). The inclusion criteria were as follows: healthy cases aged 4 - 65 years, no treatment with anti-malaria drug, not be pregnant or body temperature above $37.5^{\circ} \mathrm{C}$. The demographic information (name, age, sex, occupation, history of migration and history of malaria disease) of participants were recorded in a special form. Subjects were then evaluated for the presence or absence of clinical symptoms (specific or nonspecific). Afterwards, $2 \mathrm{~mL}$ of blood was taken from included participant and placed in sterile tubes containing $50 \mu \mathrm{L}$ of 50 mM EDTA.

Table 1. Descriptive Statistics of Asymptomatic Cases of Malaria Among Immigrants According to Gender and Age

\begin{tabular}{|cc|}
\hline Study Subjects & Subject $(\%)$ \\
\hline Age group (year) & \\
$<15$ & $30(9.1)$ \\
$15-30$ & $204(61.8)$ \\
\hline $30-45$ & $59(17.8)$ \\
$>45$ & $37(11.3)$ \\
\hline Gender & \\
\hline Male & $269(81.5)$ \\
\hline Female & $61(18.5)$ \\
\hline Total & $330(100)$ \\
\hline
\end{tabular}

\subsection{Diagnostic Tests}

\subsubsection{Microscopic Examination}

Microscopic diagnosis of malaria was conducted according to the method developed by WHO (19). Conventional microscopic examination is the gold standard method for detection of malaria parasites in suspected samples. Firstly, blood samples were taken from each participant. Secondly, some drops of blood were spread on the slide to form thin and thick smears and then smears were air-dried and fixed by methanol. Finally, fixed samples were stained with Giemsa stain and examined by oil immersion microscope lens in magnitude of 1000x. Because of low parasitemia in asymptomatic patients, all films were checked for 200 microscopic fields in about 40 $\min$. 


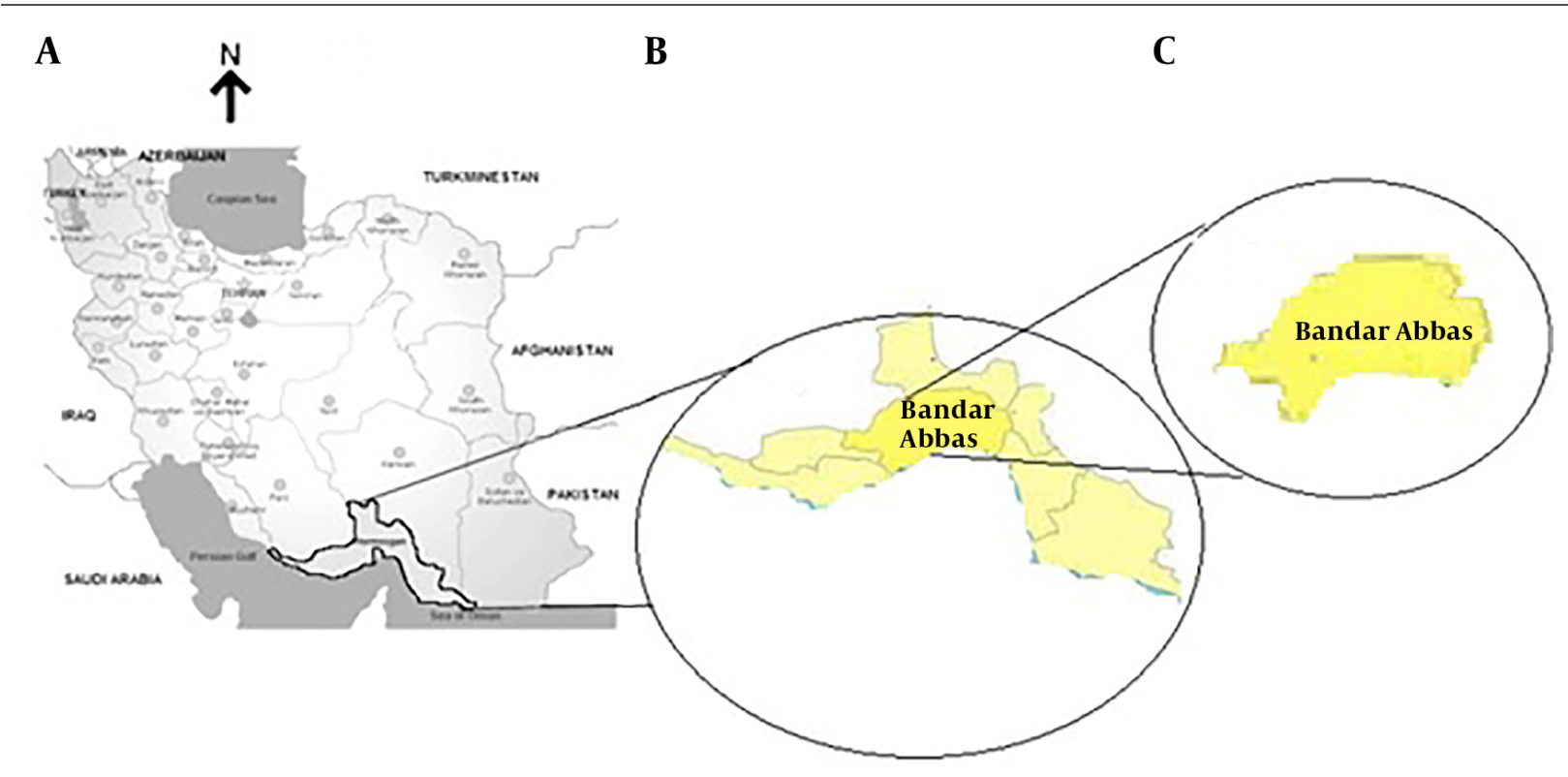

Figure 1. Iran map indicating the study area; Hormozgan province, Bandar Abbas district (https://www.google.com/search?q= Bandar Abbas district\&rlz)

\subsubsection{Rapid Diagnostic Tests}

All specimens were examined by RDTs kit along with microscopy test. The Combo test kit (Premier Medical Corporation Ltd., Mumbai, India) was used for this aim. This kit is based on antigen detection with three bands on it: one for control and others for parasite detection. To perform RDTs tests, five micro-liters (or one drop of sample) of the finger-pricked collected blood was dropped into the well of RDTs kit and the sample was run by buffers. Appearance of two or three marked lines after 20 min including control and parasite bands, indicates positive results. All the steps provided in the manufacturer's instructions were followed to do RDT test (20).

\subsubsection{Nested PCR}

Genomic DNA was isolated from whole blood using Yekta Tajhiz genomic DNA purification kit (Yekta Tajhiz, Iran). In the molecular method, the identification of Plasmodium species was performed by amplification of $18 \mathrm{sr}-$ RNA using Nested-PCR as described in previous studies (21). We utilized three kinds of primers to establish our diagnosis. The first one that amplified a 1200bp product (first PCR), was applied for detection of Plasmodium sp. The other primers amplified $120 \mathrm{bp}$ and 205bp products (second PCR) that were applied to diagnose P. vivax and P. falciparum, respectively. All PCR reactions were done in a total volume of $25 \mu \mathrm{L}$. In brief, PCR mixture contained 100 ng of purified template DNA, $250 \mathrm{nM}$ of each forward and reverse primers, $0.1 \mathrm{mM}$ of each deoxynucleotide triphosphate, $1 \times$
PCR buffer (50 mM KCl and $10 \mathrm{mM}$ tris- $\mathrm{HCl}$ ), $2.0 \mathrm{mM} \mathrm{MgCl}_{2}$, and 1.25 U Taq polymerase (Yekta Tajhiz Company, Iran).

Then, the mixture was placed in a thermocycler apparatus. PCR was performed according to predetermined program. The product of the first PCR was used as a DNA template for the second PCR. This template was mixed with reaction components and specific primers of Falciparum and Vivax and PCR was run according to the protocol introduced by Snounou et al. (21). The amplified products of the second PCR were separated on $2 \%$ agarose gel and visualized by Gel Red stain under UV illumination (Figure 2). Positive and negative controls were used in each series of PCR assay experiments. The positive control was the DNA extracted from the blood of malaria patients, which was confirmed microscopically. Negative controls were DNA extracted from the blood of healthy individuals who had no history of malaria infection and no immigration to endemic areas of malaria.

\section{Results}

The results of demographic characteristics of subjects participating in this study are shown in Table 1. Of the total 330 studied cases, $81.5 \%$ were male and $18.5 \%$ were female. The age range of subjects was 4 to 65 years and their mean age was 23.6 years.

Examination of malaria parasites using microscopic and RDT methods showed no positive cases among participants. It should be noted that microscopic tests were done 


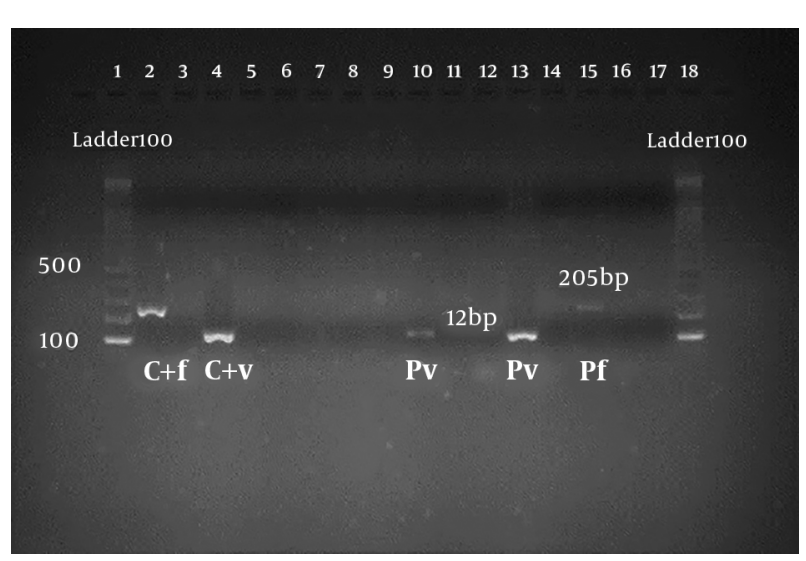

Figure 2. The gel electrophoresis results of Nested-PCR assay; ladder (lanes 1 and 18), positive control of P. falciparum (lane 2), positive control of P. vivax (lane 4), positive $P$. vivax (lanes 10 and 13), positive P. falciparum (lane 15) and negative controls (lanes 9 and 16)

by double blind microscopy experts. Examination of samples using molecular method showed five positive cases (Table 2). Four of five positive cases were found to be P. vivax and one of them was shown to be P. falciparum (Table 3).

\begin{tabular}{|c|c|}
\hline Method & Subject (\%) \\
\hline \multicolumn{2}{|l|}{ Microscopic } \\
\hline Positive & $0(0)$ \\
\hline Negative & $330(100)$ \\
\hline \multicolumn{2}{|l|}{ RDT } \\
\hline Positive & $0(0)$ \\
\hline Negative & $330(100)$ \\
\hline \multicolumn{2}{|l|}{ Molecular } \\
\hline Positive & $5(1.5)$ \\
\hline Negative & $325(98.5)$ \\
\hline Total & $330(100)$ \\
\hline
\end{tabular}

\section{Discussion}

To the best of our knowledge, this study as a part of a large-scale research on malaria elimination program in Iran, for the first time monitors asymptomatic malaria cases in immigrants in the Hormozgan province as the malaria endemic area. In order to implement the elimination program successfully, detection and monitoring of asymptomatic cases, especially in migrants from countries
Table 3. Descriptive Statistics of Asymptomatic Cases of Malaria Among Immigrants According to Parasite

\begin{tabular}{|cc|}
\hline \multicolumn{1}{l|}{ Species } & Subject (\%) \\
\hline Plasmodium vivax & \\
Positive & $4(1.22)$ \\
Negative & $326(98.78)$ \\
\hline P.falciparum & \\
\hline Positive & $1(0.31)$ \\
Negative & $329(99.69)$ \\
\hline Total & $330(100)$ \\
\hline
\end{tabular}

with a high incidence of malaria, is crucial necessity. To achieve the reliable results, in addition to the microscopic and RDT detection methods, a more robust and sensitive diagnostic technique, including tools to enable detection of parasite carriers (low parasitism, asymptomatic infections) are required. Therefore, in this study, in addition to the microscopy and serology, the molecular method was applied.

Although all sample detections were negative by microscopic and RDT method, molecular technique indicated positive cases among Afghan immigrants in Iran. Our results are in agreement with previous studies conducted in Iran. In a study by Nateghpour et al. on Afghan refugees in Sistan and Baluchestan province, the rate of asymptomatic malaria cases was reported to be $1.6 \%$ (13). It is necessary to note that in our study, the positive cases were detected using the molecular method, while in the study by Nateghpour et al. the positive cases were resulted from microscopic method. Amirshekari et al. investigated asymptomatic malaria cases using molecular technique among Pakistani and Afghan refugees in Kerman province. They reported positive cases among refugees from the both nationalities.

The results of the present study are in agreement with those conducted in US and Russia. Rodrigues et al. examined the role of African and East Asian migrants in the transmission of malaria to the United States. They reported the contribution of these immigrants to the transmission of malaria to the United States (17). Also, in another study by Baranova et al. in Russia, the role of immigrants to transmit malaria to inhabitants was confirmed (15). It should be noted that the same method as the current study was employed in these studies. In a study conducted by Rainova et al. in Bulgaria, the role of immigrants in malaria transmission after receiving a malaria elimination certificate from the WHO was examined. They showed that immigrants can still be the reservoir of malaria even after receiving a certificate (16). Therefore, obtaining a certificate cannot guaran- 
tee that malaria elimination is fully completed and monitoring programs of migrants must be continued.

In another study by Mohon et al. in Canada, they examined the diagnosis of malaria among immigrants using different diagnostic methods similar to those applied in the current study. Similar to the present study, positive cases of malaria have also been reported among immigrants in this study (18). The main advantage of this study lies in this fact that the sensitivity of the diagnosis is increased using a sensitive molecular method along with microscopic and RDT methods. This study is facing this limitation that it was not possible to follow the cases due to them changing their residence. The migration from neighboring malarious endemic countries to Iran and the relocation of these individuals has posed a major challenge to the malaria elimination program in Hormozgan province. Therefore, to achieve the goals of the malaria elimination program, more precise diagnostic technique and effective malaria surveillance actions are needed to recognize immigrant asymptomatic cases.

\subsection{Conclusions}

The results of this study are indicative of a big challenge to the Malaria Elimination Program posed by presence of asymptomatic cases migrated from malaria endemic countries in Hormozgan. In addition, this study showed that it is important to use sensitive molecular techniques along with microscopic and RDT methods to detect low parasitemia and asymptomatic cases. Further studies are suggested to be conducted in malaria high-risk areas of the Hormozgan province to monitor and evaluate asymptomatic malaria cases, especially those from other countries, in accordance with malaria elimination program.

\section{Footnotes}

Authors' Contribution: Study concept and design: Habibollah Turki and Golsoom Rashid; analysis and interpretation of data: Habibollah Turki and Aboozar Soltani; drafting of the manuscript: Habibollah Turki, Golsoom Rashid, Ebrahim Eftekhar, and Reza Shafiei; critical revision of the manuscript for important intellectual content: Habibollah Turki, Aboozar Soltani; Golsoom Rashid, and Ebrahim Eftekhar; statistical analysis: Aboozar Soltani and Reza Shafiei; acquisition of data: Golsoom Rashid and Habibollah Turki; study supervision: Habibollah Turki.

Conflict of Interests: The authors stated that there was no conflict of interest.

Ethical Approval: This study was confirmed by Ethical Committee of Hormozgan University of Medical Sciences with the ethical code of HUMS.REC.1396.40.
Funding/Support: This research was supported by the Infectious and Tropical Diseases Research Center, Hormozgan Health Institute, Hormozgan University of Medical Sciences (Technology and Research Vice-Chancellor: grant 960049).

Informed Consent: All the cases filled out the consent form.

\section{References}

1. Schapira A, Zaim M, Raeisi A, Ranjbar M, Kolifarhood G, Nikpour F, et al. History of the successful struggle against malaria in the Islamic Republic of Iran. Tehran: Neekpey; 2018.

2. Shabani SH, Zakeri S, Mehrizi AA, Mortazavi Y, Djadid ND. Population genetics structure of Plasmodium vivax circumsporozoite protein during the elimination process in low and unstable malaria transmission areas, southeast of Iran. Acta Trop. 2016;160:23-34. doi: 10.1016/j.actatropica.2016.04.006. [PubMed: 27102931].

3. Kandelaki G, Butsashvili M, Kalandadze I, Iosava M, Avaliani N, Imnadze P. Elimination of malaria in country Georgia. Acta Trop. 2012;123(1):47-8. doi: 10.1016/j.actatropica.2012.02.068. [PubMed: 22410541].

4. WHO. World Health Organization (WHO) Malaria Report 2018. 2018.

5. Nateghpour M, Tourki H, Keshavarz H, Edrisian GH, Mohebali M, Rahimi Foroushani A. A parasitological and serological study in malaria suspected patients in Hormozgan province, southeastern Iran. Iran Red Crescent Med J. 2010;12(3):242-6.

6. Turki H, Soltani A. Follow-up and monitoring of malaria treated cases toward malaria elimination program in Bashagard District, Hormozgan Province, Iran, in 2016. Jundishapur J Microbiol. 2019;12(2). e85267. doi: $10.5812 / j j m .85267$.

7. Turki H, Raeisi A, Malekzadeh K, Ghanbarnejad A, Zoghi S, Yeryan M, et al. Efficiency of nested-PCR in detecting asymptomatic cases toward malaria elimination program in an endemic area of Iran. Iran J Parasitol. 2015;10(1):39-45. [PubMed: 25904944]. [PubMed Central: PMC4403538].

8. Ebrahimzadeh A, Nouri Dalir S, Mirahmadi H, Mehravaran A, Salimi Khorashad A, Turki H. The incidence of current infection with different human malaria species by polymerase chain reaction for diagnosis of suspicious malaria patients on elimination region Sistan and Baluchistan Province, Southeast of Iran. Jundishapur J Microbiol. 2017;10(10). doi: 10.5812/jjm.58254.

9. Mirahmadi H, Spotin A, Fallahi S, Taghipour N, Turki H, Seyyed Tabaei SJ. Cloning and sequence analysis of recombinant plasmodium vivax merozoite surface protein 1 (PvMSP-142 kDa) In pTZ57R/T vector. Iran J Parasitol. 2015;10(2):197-205. [PubMed: 26246817]. [PubMed Central: PMC4522295].

10. Turki H, Zoghi S, Mehrizi AA, Zakeri S, Raeisi A, Khazan H, et al. Absence of asymptomatic malaria infection in endemic area of Bashagard District, Hormozgan Province, Iran. Iran J Parasitol. 2012;7(1):3644. [PubMed: 23133470]. [PubMed Central: PMC3488819].

11. Turki H, Rashid G, Shekari M, Raeisi A, Sharifi-Sarasiabi K. Malaria Elimination Program: Absence of asymptomatic malaria and low parasitic in endemic area of Rudan district, Hormozgan Province, Iran. Hormozgan Med J. 2017;21(4):225-31. doi: 10.29252/hmj.21.4.225.

12. Zoghi S, Mehrizi AA, Raeisi A, Haghdoost AA, Turki H, Safari R, et al. Survey for asymptomatic malaria cases in low transmission settings of Iran under elimination programme. Malar J. 2012;11:126. doi: 10.1186/1475-2875-11-126. [PubMed: 22533733]. [PubMed Central: PMC3464154].

13. Nateghpour M, Akbarzadeh K, Farivar L, Amiri A. Detection of asymptomatic malaria infection among the Afghani immigrant popula- 
tion in Iranshahr district of southeastern Iran. Bull Soc Pathol Exot. 2011;104(4):321-3. doi: 10.1007/s13149-011-0134-8. [PubMed: 21312079].

14. Amirshekari MB, Nateghpour M, Raeisi A, Motevalli Haghi A, Farivar L, Edrissian G. Determination of asymptomatic malaria among Afghani and Pakistani immigrants and native population in South of Kerman Province, Iran. Iran J Parasitol. 2016;11(2):247-52. [PubMed: 28096860]. [PubMed Central: PMC5236103].

15. Baranova A, Sergiev V, Morozova L, Turbabina N, Morozov E. Imported plasmodium vivax malaria in the Russian federation from Western Sub-Saharan Africa. J Trop Med. 2019;2019:4610498. doi: 10.1155/2019/4610498. [PubMed: 30936925]. [PubMed Central: PMC6413362].

16. Rainova IG, Harizanov RN, Kaftandjiev IT, Mikov OD, Tsvetkova ND. Imported malaria in Bulgaria, status and prognosis after eradication in 1965. J Infect Public Health. 2018;11(4):534-9. doi: 10.1016/j.jiph.2017.10.010. [PubMed: 29126828].

17. Rodrigues PT, Valdivia HO, de Oliveira TC, Alves JMP, Duarte A, CeruttiJunior C, et al. Human migration and the spread of malaria parasites to the New World. Sci Rep. 2018;8(1):1993. doi:10.1038/s41598-018-195540. [PubMed: 29386521]. [PubMed Central: PMC5792595].

18. Mohon AN, Lee LD, Bayih AG, Folefoc A, Guelig D, Burton RA, et al. NINALAMP compared to microscopy, RDT, and nested PCR for the detection of imported malaria. Diagn Microbiol Infect Dis. 2016;85(2):149-53. doi: 10.1016/j.diagmicrobio.2015.11.009. [PubMed: 27017271]. [PubMed Central: PMC4862928].

19. WHO. Malaria microscopy quality assurance manual-version 2: World Health Organization. 2016.

20. Maltha J, Gillet P, Jacobs J. Malaria rapid diagnostic tests in endemic settings. Clin Microbiol Infect. 2013;19(5):399-407. doi: 10.1111/14690691.12151. [PubMed: 23438048].

21. Snounou G, Viriyakosol S, Jarra W, Thaithong S, Brown KN. Identification of the four human malaria parasite species in field samples by the polymerase chain reaction and detection of a high prevalence of mixed infections. Mol Biochem Parasitol. 1993;58(2):283-92. doi: 10.1016/0166-6851(93)90050-8. [PubMed: 8479452]. 